九州大学学術情報リポジトリ

Kyushu University Institutional Repository

\title{
ASYMPTOTIC NORMALITY OF RANK SUMS UNDER DEPENDENCY AND ITS APPLICATIONS TO THE TESTING PROBLEM
}

Tamura, Ryoj i

Department of Mathematics, Faculty of Science, Kumamoto University

https://doi.org/10.5109/13143

出版情報: 統計数理研究. 19 (3/4), pp.1-8, 1981-03. Research Association of Statistical Sciences

バージョン：

権利関係 : 


\title{
ASYMPTOTIC NORMALITY OF RANK SUMS UNDER DEPENDENCY AND ITS APPLICATIONS TO THE TESTING PROBLEM
}

\author{
By
}

\author{
Ryoji TAMURA*
}

(Received March 7, 1980)

\section{Introduction.}

Let the distribution function of the random vector $\boldsymbol{X}=\left(X_{1}, \cdots, X_{c}\right)$ be $H\left(x_{1}, \cdots, x_{c}\right)$ and the marginal distribution function of $X_{i}$ be $F_{i}(x), i=1, \cdots, c$ where we assume $H$ to be absolutely continuous. Further, let $\boldsymbol{X}_{\alpha}=\left(X_{1 \alpha}, \cdots, X_{c \alpha}\right), \alpha=1, \cdots, n$ be a random sample from the population with the distribution function $H$. Now we denote the rank of $X_{i \alpha}$ among nc random variables $\left\{X_{i \alpha}, i=1, \cdots, c, \alpha=1, \cdots, n\right\}$ by $R_{i \alpha}$ and define $R_{i}=\sum_{\alpha=1}^{n} R_{i \alpha} / n(n-1)$ for $i=1, \cdots, c$.

Then this paper is concerned with the asymptotic distribution of the linear rank statistic $R_{d}=\sqrt{n} \sum_{i=1}^{c} d_{i}\left(R_{i}-p_{i}\right)$ and its applications to some testing problem where $d_{i}$ 's are any constants which are not all equal and $p_{i}$ 's are defined in section 2 . The asymptotic distribution of rank statistics, which is one of the most essential parts in nonparametric theory, has been studied by many workers and in particular, it is wellknown that Chernoff-Savage [1] and Hájek [2] have established most fruitful results in this field. They have discussed under the assumption that the compononts $X_{i}$ 's are independent, but not considered for the case that the independence of $X_{i}$ 's is violated. It is, therefore, of interest in studying the asymptotic distribution of $R_{d}$ and its applications when $X_{i}$ 's are not independent, though $R_{d}$ is the simplest rank statistic.

In what follows, the summation $\Sigma$ extends over all integers from 1 to $c$ when the index is $i, j, k$ or $l$ and from 1 to $n$ when the index is $\alpha, \beta, \gamma$ or $\delta$.

\section{Asymptotic normality of $R_{d}$.}

The rank $R_{i \alpha}$ of $X_{i \alpha}$ may be written as follows,

$$
R_{i \alpha}=\sum_{j} \sum_{\beta} u\left(X_{i \alpha}-X_{j \beta}\right)+1
$$

where

* Department of Mathematics, Faculty of Science, Kumamoto University, Kumamoto. 
Then we easily obtain

$$
u(x)= \begin{cases}1 & \text { for } x>0 \\ 0 & \text { otherwise }\end{cases}
$$

$$
\begin{aligned}
& E\left(R_{i \alpha}\right)=(n-1) p_{i}+p_{1}^{\prime}+1 \\
& E\left(R_{i}\right)=p_{i}+\left(p_{i}^{\prime}+1\right) /(n-1),
\end{aligned}
$$

where

$$
p_{i}=\sum_{j} p_{i j}, \quad p_{i j}=E\left(F_{j}\left(X_{i}\right)\right), \quad p_{i}^{\prime}=\sum_{j} p_{i j}^{\prime}, \quad p_{i j}^{\prime}=P\left(X_{i}>X_{j}\right)
$$

and

$$
p_{i j}=1-p_{j i}, \quad p_{i j}=1 / 2 \text { if } F_{i}=F_{j}, \quad p_{i i}^{\prime}=0 .
$$

Now let $\approx$ be the class of statistics $L$ of the form $L=\sum_{\alpha} k_{\alpha}\left(\boldsymbol{X}_{\alpha}\right)$ where the functions $k_{\alpha}$ may be chosen arbitrarily except $E\left(k_{a k}^{2}\left(\boldsymbol{X}_{\alpha}\right)\right)<\infty$. We shall first consider the projection of $R_{i}$ on the space $\stackrel{\Omega}{\text {. }}$

2.1. The projection of $R_{i}$ on $\mathbb{R}$. We denote the projection of $R_{i}$ on $\mathbb{Z}$ by $T_{i}$. Then we get the following by the multivariate form of the projection lemma due to Hájek [2]

$$
\begin{aligned}
T_{i} & =\sum_{\alpha} E\left(R_{i} \mid X_{\alpha}\right)-(n-1) E\left(R_{i}\right) \\
E\left(T_{i}\right) & =E\left(R_{i}\right), \quad E\left(R_{i}-T_{i}\right)^{2}=\operatorname{Var}\left(R_{i}\right)-\operatorname{Var}\left(T_{i}\right) .
\end{aligned}
$$

We may first obtain after some calculations,

$$
E\left(R_{i, \hat{\beta}} \mid \boldsymbol{X}_{\alpha}\right)= \begin{cases}\sum_{j}\left[(n-1) F_{j}\left(X_{i \alpha}\right)+u\left(X_{i \alpha}-X_{j \hat{\beta}}\right)\right]+1 & \beta=\alpha \\ \sum_{j}\left[\left(1-F_{i}\left(X_{j \alpha}\right)\right)+(n-2) p_{i j}+p_{i j}^{\prime}\right]+1 & \beta \neq \alpha\end{cases}
$$

and hence

$$
\begin{aligned}
E\left(R_{i} \mid \boldsymbol{X}_{\alpha}\right)= & \frac{1}{n} \sum_{j}\left[F_{j}\left(X_{i \alpha}\right)+1-F_{i}\left(X_{j \alpha}\right)\right]+\frac{n-2}{n} p_{i} \\
& +\frac{1}{n} p_{i}^{\prime}+\frac{1}{n(n-1)} \sum_{j} u\left(X_{i \alpha}-X_{j \alpha}\right)+\frac{1}{n-1}
\end{aligned}
$$

From (2.4) and (2.5), we also obtain

$$
\begin{aligned}
T_{i}-E\left(T_{i}\right)= & \frac{1}{n} \sum_{\alpha} \sum_{j}\left[F_{j}\left(X_{i \alpha}\right)+1-F_{i}\left(X_{j \alpha}\right)-2 p_{i j}\right] \\
& +\frac{1}{n(n-1)} \sum_{\alpha} \sum_{j} u\left(X_{i \alpha}-X_{j \alpha}\right)-\frac{1}{n-1} p_{i}^{\prime} .
\end{aligned}
$$

We shall show in Lemma 2.1 that $R_{i}$ is asymptotically equivalent to its projection $T_{i}$.

LEMMA 2.1.

$$
\sqrt{n}\left(R_{i}-T_{i}\right) \stackrel{P}{\longrightarrow} 0 \quad \text { as } n \rightarrow \infty
$$


Proof. Since it follows from (2.4) that

$$
n E\left(R_{i}-T_{i}\right)^{2}=n \operatorname{Var}\left(R_{i}\right)-n \operatorname{Var}\left(T_{i}\right), \quad E\left(R_{i}\right)=E\left(T_{i}\right),
$$

if suffices to show that

$$
n\left(\operatorname{Var}\left(R_{i}\right)-\operatorname{Var}\left(T_{i}\right)\right) \longrightarrow 0 \quad \text { as } n \rightarrow \infty .
$$

Obviously,

$$
n \operatorname{Var}\left(R_{i}\right)=\frac{1}{n(n-1)^{2}}\left[\sum_{\alpha} \operatorname{Var}\left(R_{i \alpha}\right)+\sum_{\alpha \neq \beta} \sum_{\beta} \operatorname{Cov}\left(R_{i \alpha}, R_{i_{\beta},}\right)\right] .
$$

After somewhat complicated calculations, we can get

$$
\operatorname{Var}\left(R_{i \alpha}\right)=(n-1)(n-2) \sum_{k} \sum_{l}\left[E\left(F_{k}\left(X_{i}\right) F_{l}\left(X_{i}\right)\right)-p_{i k} p_{i l}\right]+O(n)
$$

and for $\alpha \neq \beta$,

$$
\begin{aligned}
\operatorname{Cov}\left(R_{i \alpha}, R_{i ;}\right)= & (n-2) \sum_{k} \sum_{l}\left[E\left\{F_{l}\left(X_{i}\right)\left(1-F_{i}\left(X_{k}\right)\right)\right\}+E\left\{F_{k}\left(X_{i}\right)\left(1-F_{i}\left(X_{l}\right)\right)\right\}\right. \\
& \left.+E\left\{\left(1-F_{i}\left(X_{k}\right)\right)\left(1-F_{i}\left(X_{l}\right)\right)\right\}-3 p_{i k} p_{i l}\right]+O(1),
\end{aligned}
$$

and consequently.

$$
\begin{aligned}
n \operatorname{Var}\left(R_{i}\right)= & \sum_{k} \sum_{l} E\left[\left(F_{k}\left(X_{i}\right)+1-F_{i}\left(X_{k}\right)-2 p_{i k}\right)\right. \\
& \left.\cdot\left(F_{l}\left(X_{i}\right)+1-F_{i}\left(X_{l}\right)-2 p_{i l}\right)\right]+O\left(\frac{1}{n}\right) .
\end{aligned}
$$

As for $\operatorname{Var}\left(T_{i}\right)$, a direct calculation shows that

$$
\begin{aligned}
n \operatorname{Var}\left(T_{i}\right) & =\frac{1}{n} \operatorname{Var}\left(\sum_{\alpha} \sum_{j}\left[F_{j}\left(X_{i \alpha}\right)+1-F_{i}\left(X_{j \alpha}\right)-2 p_{i j}\right]+O\left(\frac{1}{n}\right)\right. \\
& =\sum_{k} \sum_{l} E\left[\left(F_{k}\left(X_{i}\right)+1-F_{i}\left(X_{k}\right)-2 p_{i k}\right)\left(F_{l}\left(X_{i}\right)+1-F_{i}\left(X_{l}\right)-2 p_{i l}\right)\right]+O\left(\frac{1}{n}\right) .
\end{aligned}
$$

Thus (2.7) easily follows from (2.8) and (2.9).

2.2. Asymptotic normality of $\sqrt{n} \sum_{i} d_{i}\left(R_{i}-p_{i}\right)$.

LEMMA 2.2.

$$
\sqrt{n} \sum_{i} d_{i}\left(R_{i}-p_{i}\right)-\sqrt{n} \sum_{i} d_{i} \hat{T}_{i} \stackrel{P}{\longrightarrow} 0 \quad \text { as } n \rightarrow \infty,
$$

where

$$
\hat{T}_{i}=\frac{1}{n} \sum_{\alpha} \sum_{j}\left[F_{j}\left(X_{i \alpha}\right)+1-F_{i}\left(X_{j \alpha}\right)-2 p_{i j}\right] .
$$

Proof. It is obvious from (2.6) and (2.3) that

$$
\sqrt{n}\left(T_{i}-E\left(T_{i}\right)-\hat{T}_{i}\right)=\sum_{\alpha} \sum_{j}\left(u\left(X_{i \alpha}-X_{j \alpha}\right)-p_{i j}^{\prime}\right) \stackrel{P}{\longrightarrow} 0,
$$




$$
\sqrt{n}\left(R_{i}-p_{i}-\left(R_{i}-E\left(R_{i}\right)\right)=\frac{\sqrt{n}}{n-1}\left(p_{i}^{\prime}+1\right) \longrightarrow 0 .\right.
$$

From these results and Lemma 2.1, we have

$$
\begin{aligned}
\sqrt{n} \sum_{i} d_{i}\left(R_{i}-p_{i}-\hat{T}_{i}\right)= & \sqrt{n} \sum_{i} d_{i}\left[\left(R_{i}-p_{i}-\left(R_{i}-E\left(R_{i}\right)+\left(R_{i}-T_{i}\right)\right.\right.\right. \\
& +\left(T_{i}-E\left(T_{i}\right)-\hat{T}_{i}\right] \stackrel{P}{\longrightarrow} 0 .
\end{aligned}
$$

THEOREM 2.1. $\sqrt{n} \sum_{i} d_{i}\left(R_{i}-p_{i}\right)$ has the limitting normal distribution $N\left(0, \sigma^{2}\left(R_{d}\right)\right)$ provided that $\sigma^{2}\left(R_{d}\right) \neq 0$, where

$$
\sigma^{2}\left(R_{d}\right)=\operatorname{Var}\left(\sum_{i} \sum_{j} d_{i}\left(F_{j}\left(X_{i}\right)-F_{i}\left(X_{j}\right)\right)\right) .
$$

ProOF. From Lemma 2.2, it suffices to show the asymptotic normality of $\sqrt{n} \sum_{i} d_{i} \hat{T}_{i}$. Now we may express $\sum_{i} d_{i} \hat{T}_{i}$ by

$$
\sum_{i} d_{i} \hat{T}_{i}=\frac{1}{n} \sum_{\alpha} Y_{\alpha}, \quad \text { where } \quad Y_{\alpha}=\sum_{i} \sum_{j} d_{i}\left[F_{j}\left(X_{i \alpha}\right)+1-F_{i}\left(X_{j \alpha}\right)-2 p_{i j}\right] .
$$

Then $\left\{Y_{\alpha}\right\}, \alpha=1, \cdots, n$ are independent and identically distributed random variables with $\operatorname{Var}\left(V_{\alpha}\right)<\infty$. Thus we may apply the central limit theorem to show the asymptotic normality $N(0,1)$ of $\sqrt{n} \sum_{i} d_{i} \hat{T}_{i} / \sigma\left(R_{d}\right)$ if $\sigma\left(R_{d}\right) \neq 0$.

2.3. A consistent estimator of $\sigma^{2}\left(R_{d}\right)$. In order to use $R_{d}$ for the testing problem in section 3 , we here derive a consistent estimator of $\sigma^{2}\left(R_{d}\right)$. Define $S_{\alpha}, \alpha=1, \cdots, n$ as follows,

$$
\begin{aligned}
& S_{\alpha}=\frac{1}{n} \sum_{i} \sum_{j} \sum_{\beta} d_{i}\left[u\left(X_{i \alpha}-X_{j \beta}\right)+u\left(X_{i \beta}-X_{j \alpha}\right)\right] \\
& \hat{\sigma}_{n}^{2}=\frac{1}{n} \sum_{\alpha}\left(S_{\alpha}-\bar{S}\right)^{2}, \quad \text { where } \quad \bar{S}=\frac{1}{n} S_{\alpha} .
\end{aligned}
$$

LEMMA 2.3. $\hat{\sigma}_{\bar{n}}^{\circ}$ is a consistent estimator of $\sigma^{2}\left(R_{d}\right)$.

Proof. Notice first that $\left\{S_{\alpha}\right\}, \alpha=1, \cdots, n$ are independent and identically distributed random variobles. $S_{\alpha}^{2}$ may be expressed as

$$
S_{\alpha}^{2}=\frac{1}{n^{2}} \sum_{i} \sum_{j} \sum_{k} \sum_{l} \sum_{\beta} \sum_{\gamma} d_{i} d_{j} v_{i k \alpha \beta} v_{j l \alpha \gamma},
$$

where

Then we have

$$
v_{i k \alpha \beta}=u\left(X_{i \alpha}-X_{k \beta}\right)+u\left(X_{i \beta}-X_{k \alpha}\right) .
$$

$$
E\left(S_{\alpha}^{2}\right)=\sum_{i} \sum_{j} \sum_{k} \sum_{l} d_{i} d_{j} E\left[\left(F_{k}\left(X_{i}\right)+1-F_{i}\left(X_{k}\right)\right)\left(F_{l}\left(X_{j}\right)+1-F_{j}\left(X_{l}\right)\right)\right]+O\left(\frac{1}{n}\right) .
$$

Since $\bar{S}$ may be written as 


$$
\bar{S}=\frac{1}{n^{2}} \sum_{i} \sum_{j} \sum_{\alpha} \sum_{\beta} d_{i} v_{i j \alpha \beta}=\frac{2(n-1)}{n} \sum_{i} d_{i} R_{i}
$$

we get from (2.3)

$$
E(\bar{S})=2 \sum_{i} d_{i} p_{i}+O\left(\frac{1}{n}\right)
$$

Therefore we easily find from (2.14) and (2.15) that

$$
E\left(\hat{\sigma}_{n}^{2}\right)=\sum_{i} \sum_{j} \sum_{k} \sum_{l} d_{i} d_{j} E\left[\left(F_{k}\left(X_{i}\right)+1-F_{i}\left(X_{k}\right)-2 p_{i k}\right)\left(F_{l}\left(X_{j}\right)+1-F_{j}\left(X_{l}\right)-2 p_{j l}\right)\right]+O\left(\frac{1}{n}\right)
$$

$$
=\operatorname{Var}\left[\sum_{i} \sum_{j} d_{i}\left(F_{j}\left(X_{i}\right)-F_{i}\left(X_{j}\right)\right)\right]+O\left(\frac{1}{n}\right) \longrightarrow \sigma^{2}\left(R_{d}\right) \quad \text { as } \quad n \rightarrow \infty .
$$

We shall turn to show that $\operatorname{Var}\left(\hat{\sigma}_{n}^{2}\right) \rightarrow 0$ as $n \rightarrow \infty$. Since $\hat{\sigma}_{n}^{2}$ may be written as

$$
\hat{\sigma}_{n}^{2}=\frac{1}{n^{3}} \sum_{\alpha}\left(\sum_{\beta} v_{\alpha \beta}\right)^{2}-\frac{1}{n^{4}}\left(\sum_{\alpha} \sum_{\beta} v_{\alpha \beta}\right)^{2}, \quad \text { where } v_{\alpha \beta}=\sum_{i} \sum_{j} d_{i} v_{i j \alpha \beta},
$$

we easily find the following

$$
\operatorname{Var}\left(\hat{\sigma}_{n}^{2}\right)=\frac{1}{n^{6}} \operatorname{Var}\left(\sum_{\alpha} \sum_{\beta} \sum_{\gamma} v_{\alpha \beta} v_{\alpha \gamma}\right)+\frac{1}{n^{8}} \operatorname{Var}\left(\sum_{\alpha} \sum_{\beta} \sum_{\gamma} \sum_{\delta} v_{\alpha \beta} v_{\gamma \bar{o}}\right)
$$

$$
-\frac{2}{n^{7}} \operatorname{Cov}\left(\sum_{\alpha} \sum_{\beta} \sum_{\gamma} v_{\alpha \beta} v_{\alpha \gamma}, \sum_{\alpha} \sum_{\beta} \sum_{\gamma} \sum_{\delta} v_{\alpha \beta} v_{\gamma j}\right)
$$

After some elementary but tedious calculations, it follows that

$$
\begin{aligned}
& \operatorname{Var}\left(\sum_{\alpha} \sum_{\beta} \sum_{\gamma} v_{\alpha \beta} v_{\alpha \gamma}\right)=O\left(n^{5}\right) \\
& \operatorname{Var}\left(\sum_{\alpha} \sum_{\beta} \sum_{\gamma} \sum_{\delta} v_{\alpha \beta} v_{\gamma \delta}\right)=O\left(n^{7}\right) \\
& \operatorname{Cov}\left(\sum_{\alpha} \sum_{\beta} \sum_{\gamma} v_{\alpha \beta} v_{\alpha \gamma}, \sum_{\alpha} \sum_{\beta} \sum_{\gamma} \sum_{\delta} v_{\alpha \beta} v_{\gamma \delta}\right)=O\left(n^{6}\right) .
\end{aligned}
$$

Thus we obtain from (2.17) and (2.18)

$$
\operatorname{Var}\left(\hat{\sigma}_{n}^{2}\right) \longrightarrow 0 \quad \text { as } n \rightarrow \infty \text {. }
$$

Lemma 2.3 follows from (2.16) and (2.19).

COROLLARY 2.1. The statistic $\sqrt{n} \Sigma d_{i}\left(R_{i}-p_{i}\right) / \hat{\sigma}_{n}$ has the limiting standard normal distribution.

The proof is easily shown from Theorem 2.1 and Lemma 2.3.

\section{A class of tests for homogeneity of marginal distributions.}

As an application of the results in section 2, we here consider the problem of testing hypothesis 


$$
H_{0}: F_{1}(x)=\cdots=F_{c}(x)
$$

against the ordered alternative

$$
H_{1}: F_{1}(x) \geqq \cdots \geqq F_{c}(x),
$$

where at least one of the inequalities is strict.

For this problem, Tamura [4] has proposed a test $W$ under that $X_{i}^{\prime}$ s are not independent, that is

$$
W=\sum_{i<j} W_{i j}, \quad W_{i j}=\sum_{\alpha \neq \beta} \sum_{\alpha} u\left(X_{j \beta}-X_{i \alpha}\right),
$$

where we note that $W_{i j}$ is the $U$-statistic proposed by Raviv [3] for the two-sample problem. We here propose a class of tests $R_{d}$ which reject $H_{0}$ if

$$
\sqrt{n} \sum_{i} d_{i}\left(R_{i}-\frac{c}{2}\right) \geqq \hat{\sigma}_{n} z_{\alpha}
$$

where $d_{i}^{\prime}$ s are any constants satisfying $d_{i} \leqq \cdots \leqq d_{c}$ (at least one of inequalities is strict) and $z_{\alpha}$ is the $(1-\alpha)$ percentile point of the standard normal distribution $\Phi(x)$. Then it is easily shown from Corollary 2.1 that the test $R_{d}$ has asymptotic level $\alpha$ of significance.

It is very important in applications how to choose $d_{i}^{\prime} \mathrm{s}$, but we can get no answer for this problem. We can only show later that a test $R_{d}$ with $d_{i}=i$ for $i=1, \cdots, c$ is asymptotically equivalent to the test $W$.

3.1. Asymptotic power of $R_{d}$. We shall now derive the asymptotic power of the test $R_{d}$ for the translation alternative

$$
H_{1}^{*}: F_{i}(x)=F\left(x-\theta_{i} / \sqrt{N}\right), \quad i=1, \cdots, c,
$$

where $\theta_{1} \leqq \cdots \leqq \theta_{c}$ (at least one of inequalities is strict) and $n / N \rightarrow \lambda, 0<\lambda_{0} \leqq \lambda \leqq \lambda_{1}$, as $N \rightarrow \infty$. Denoting the limiting power function of the test $R_{d}$ be $\beta_{R}(\boldsymbol{\theta})$, then we have

$$
\begin{aligned}
\beta_{R}(\boldsymbol{\theta}) & =\lim _{N \rightarrow \infty} P\left[\sqrt{n} \sum_{i} d_{i}\left(R_{i}-\frac{c}{2}\right) \geqq \hat{\sigma}_{n} z_{\alpha} \mid H_{1}^{*}\right] \\
& =\lim _{N \rightarrow \infty} P\left[\sqrt{n} \sum_{i} d_{i}\left(R_{i}-p_{i}^{*}\right) \geqq \hat{\sigma}_{n} z_{\alpha}+\sqrt{n} \sum_{i} d_{i}\left(\frac{c}{2}-p_{i}^{*}\right) \mid H_{1}^{*}\right],
\end{aligned}
$$

where

$$
p_{i}^{*}=\sum_{j} p_{i j}^{*}, \quad p_{i j}^{*}=E\left[F\left(X_{i}-\theta_{j} / \sqrt{ } \bar{N}\right) \mid H_{1}^{*}\right] .
$$

We here notice that (i) under $H_{1}^{*}$

$$
\begin{aligned}
\hat{\sigma}_{n}^{2} \rightarrow \sigma_{0}^{2}\left(R_{d}\right) & =\operatorname{Var}\left(\sum_{i} \sum_{j} d_{i}\left(F\left(X_{i}\right)-F\left(X_{j}\right)\right) \mid H_{0}\right) \\
& =\frac{c^{2}}{12} \sum_{i} \sum_{j}\left(d_{i}-\bar{d}\right)\left(d_{j}-\bar{d}\right) \rho_{0}\left(F\left(X_{i}\right), F\left(X_{j}\right)\right),
\end{aligned}
$$

where $\rho_{0}\left(F\left(X_{i}\right), F\left(X_{j}\right)\right)$ is the correlation coefficient of $F\left(X_{i}\right)$ and $F\left(X_{j}\right)$ under $H_{0}$ and 
$\bar{d}=\sum_{i} d_{i} / c$ and (ii) under the assumption that the density $f$ of $F$ is bounded,

$$
\begin{gathered}
\sqrt{n}\left(\frac{c}{2}-p_{i}^{*}\right)=\sqrt{n} \int_{-\infty}^{\infty}\left[F\left(x+\left(\theta_{i}-\theta_{j}\right) / \sqrt{N}\right)-F(x)\right] d F(x) \\
\longrightarrow \sqrt{\lambda}\left(\theta_{i}-\theta_{j}\right) \int_{-\infty}^{\infty} f(x) d F(x) \quad \text { as } \quad N \rightarrow \infty
\end{gathered}
$$

From Corillary 2.1, (3.3) and (3.4), $\beta_{R}(\boldsymbol{\theta})$ is represented as follows,

$$
\beta_{R}(\theta)=1-\Phi\left(z-\sqrt{\lambda c}\left(\int_{-\infty}^{\infty} f^{2}(x) d x \sum_{i}\left(d_{i}-\bar{d}\right)\left(\theta_{i}-\bar{\theta}\right) / \sigma_{0}\left(R_{d}\right)\right),\right.
$$

where $\bar{\theta}=\sum_{i} \theta_{i} / c$

3.2. Comparison with other tests.

(a) The test $W$. The test $W$ reject $H_{0}$ if

$$
W \geqq \frac{1}{4} c(c-1)+\hat{A}_{n} z_{\alpha} / \sqrt{n},
$$

where $\hat{A}_{\bar{n}}^{2}$ is a consistent estimator of $A^{2}=\operatorname{Var}\left(\sum_{i<j} \sum_{j}\left[F_{j}\left(X_{i}\right)-F_{i}\left(X_{j}\right)\right]\right)$ as defined in [4]. Then the limiting power of the test $W$ under $H_{1}^{*}$ has been expressed by

$$
\beta_{W}(\boldsymbol{\theta})=1-\Phi\left(z_{\alpha}-\sqrt{\lambda}\left(\int_{-\infty}^{\infty} f^{2}(x) d x \sum_{i<j}\left(\theta_{j}-\theta_{i}\right)\right) / A_{0},\right.
$$

where

$$
A_{0}^{2}=\operatorname{Var}\left(\sum_{i<j}\left[F\left(X_{i}\right)-F\left(X_{j}\right)\right] \mid H_{o}\right) .
$$

Comparing the test $R_{d}$ when $d_{i}=i$ with the test $W$ in the limiting power, we can easily show $\beta_{R}(\boldsymbol{\theta})=\beta_{W}(\boldsymbol{\theta})$ by noticing the identities

$$
\begin{aligned}
& \sum_{i} \sum_{j} i\left(F\left(X_{i}\right)-F\left(X_{j}\right)\right)=\frac{c}{2} \sum_{i<j} \sum_{i}\left(F\left(X_{i}\right)-F\left(X_{j}\right)\right), \\
& \sum_{i} i\left(\theta_{i}-\bar{\theta}\right)=\sum_{i<j}\left(\theta_{j}-\theta_{i}\right) / 2
\end{aligned}
$$

and consequently, $\sigma_{0}^{2}\left(R_{d}\right)=c^{2} A_{0}^{2} / 4$.

(b) The test based on the sample means. Finally, we shall make large sample comparison between the test $R_{d}$ and the coresponding competitor $T_{d}$ based on Stusent statistic,

$$
T_{d}=\sqrt{n} \sum_{i} d_{i}\left(\bar{X}_{i}-\bar{X}\right), \quad \bar{X}_{i}=\sum_{\alpha} X_{i \alpha} / n, \quad \overline{\bar{X}}=\sum_{i} \bar{X}_{i} / c,
$$

This statistic will be used for our testing problem assuming that $\operatorname{Cov}(X)$ is known. Then the test $T_{d}$ of asymptotic level $\alpha$ reject $H_{0}$ if

$$
T_{d} \geqq \sigma_{0}\left(T_{d}\right) z_{\alpha}, \quad \sigma_{0}^{2}\left(T_{d}\right)=\sigma_{0}(X) \sum_{i} \sum_{j}\left(d_{i}-\bar{d}\right)\left(d_{j}-\bar{d}\right) \rho_{0}\left(X_{i}, X_{j}\right)
$$


where $\sigma_{0}^{2}(X)$ and $\rho_{0}\left(X_{i}, X_{j}\right)$ are respectively the variance of $X_{i}$ and the correlation of $X_{i}$ and $X_{j}$ under $H_{0}$.

Asymptotic normality of $T_{d}$ is easily shown as in [4] and consequently, we can get the limiting power under $H_{1}^{*}$

$$
\beta_{T}(\boldsymbol{\theta})=1-\Phi\left(z_{\alpha}-\sqrt{\lambda} \sum_{i}\left(d_{i}-\bar{d}\right)\left(\theta_{i}-\bar{\theta}\right) / \sigma_{0}\left(T_{d}\right) .\right.
$$

From (3.4) and (3.10), the Pitman relative efficiency of $R_{d}$ with respect to $T_{d}$ may be expressed as follows

$$
\begin{aligned}
e\left(R_{d}, T_{d}\right)= & 12 \sigma_{0}^{2}(X)\left(\int_{-\infty}^{\infty} f^{2}(x) d x\right)^{2} \sum_{i} \sum_{j}\left(d_{i}-\bar{d}\right)\left(d_{j}-\bar{d}\right) \rho_{0}\left(X_{i}, X_{j}\right) \\
& \div \sum_{i} \sum_{j}\left(d_{i}-\bar{d}\right)\left(d_{j}-\bar{d}\right) \rho_{0}\left(F\left(X_{i}\right), F\left(X_{j}\right)\right)
\end{aligned}
$$

\section{References}

[1] Cherenoff, H. and Savage, I. R.: Asymptotic normality and efficiency of certain nonparametric test statistics. Ann. Math. Statist. 29 (1958) 972-994.

[2] HÁJEK, J.: Asymptotic normality of simple linear rank statistics under alternatives. Ann. Math. Statist. 39 (1968) 325-346.

[3] Raviv, A.: A non-parametric test for comparing two non-independent distributions. J. Roy. Statist. Soc. B 40 (1978) 253-261.

[4] Tamura, R.: A test of Wilcoxon type for homogeneity of marginal distributions against ordered alternatives. Kumamoto J. Sci. (Math.) 14 (1980) 34-41.

Department of Mathematics

Faculty of Science

Kumamoto University

Editorial: This paper is the last publication by Professor Ryoji Tamura who died on November 13, 1980 in Kumamoto. 